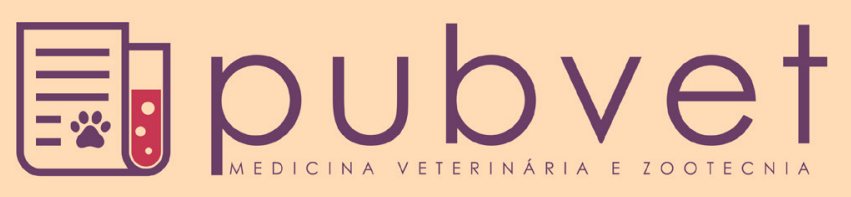

ISSN $1982-1263$

HTTP://DX.DOI.ORG/10.22256/PUBVET.VI1N5.513-518

\title{
Dinâmica do acúmulo de forragem e morfogênese de Urochloa brizantha cv. Xaraés sob períodos de descanso
} \author{
Seixas Santos 5 \\ ${ }^{I}$ Eng. Agr., D.Sc., Pesquisador da Embrapa Roraima, Boa Vista, RR. \\ ${ }^{2} Z$ Zootecnista, D.Sc., Pesquisador da Embrapa Clima Temperado, Pelotas, RS \\ ${ }^{3}$ Méd. Vet., Pesquisador da Embrapa Meio-Norte, Parnaíba, PI. \\ ${ }^{4}$ Zootecnista, D.Sc., Pesquisador da Embrapa Meio-Norte, Parnaiba, PI. \\ ${ }^{5}$ Eng. Agr., D.Sc., Pesquisador da Embrapa Meio-Norte, Parnaíba, PI. \\ *Autor para correspondência, E-mail: newtonlucena@yahoo.com.br \\ **in memorian
}

Newton de Lucena Costa ${ }^{1^{*}}$, Claudio Ramalho Townsend ${ }^{2 * *}$, João Avelar Magalhães ${ }^{3}$, Fabíola Helena dos Santos Fogaça ${ }^{4}$, Amaury Burlamaqui Bendahan ${ }^{1}$, Francisco José de

RESUMO. O efeito do período de descanso $(14,21,28,35,42$ e 49 dias) sobre a produção e composição química da forragem e características morfogênicas e estruturais de Urochloa brizantha cv. Xaraés foi avaliado em condições de campo. O aumento do período de descanso resultou em maiores rendimentos de matéria seca verde (MSV) e vigor de rebrota, contudo implicou em decréscimos significativos dos teores de nitrogênio, fósforo, cálcio, magnésio e potássio. O comprimento médio de folhas e suas taxas de expansão e senescência foram diretamente proporcionais aos períodos de descanso, ocorrendo o inverso quanto à taxa de aparecimento foliar. Os maiores rendimentos de MSV, número de folhas vivas, taxa de expansão foliar e índice de área foliar foram obtidos aos 40,8; 40,2; 26,2 e 48,2 dias de rebrota. O período de descanso mais adequado para pastagens de $U$. brizantha cv. Xaraés, visando a conciliar produção, vigor de rebrota e qualidade da forragem, situa-se entre 35 e 42 dias.

Palavras chave: Composição química, folhas, matéria seca, perfilhamento, senescência

\section{Forage dynamic accumulation and morphogenesis of Urochloa brizantha $c v$. Xaraés under rest periods}

\begin{abstract}
The effects of rest periods $(14,21,28,35,42$ and 49 days) on green dry matter (GDM) yield, chemical composition and morphogenetic and structural characteristics of Urochloa brizantha cv. Xaraés were evaluated under natural field conditions. GDM yields and regrowth, medium blade length, leaf elongation and leaf senescence rate increased consistently with highest rest periods however the nitrogen, phosphorus, calcium, magnesium and potassium contents and leaf appearance rate decreased. Maximum GDM yields, number of leaves tiller-1 ${ }^{-1}$ leaf elongation rate and leaf area index were obtained with rest periods at $40.8 ; 40.2 ; 26.2$ and 48.2 days. These data suggest that grass grazing at 35 to 42 days of rest period were optimal for obtain maximum yields and regrowth of rich forage.
\end{abstract}

Keywords: chemical composition, dry matter, leaves, tillering, senescence

Dinámica de la acumulación del forraje y morfogénesis de Urochloa brizantha cv. Xaraés bajo períodos de descanso 
RESUMEN. El efecto del período de descanso (14, 21, 28, 35, 42 y 49 dias) sobre la producción y la composición química del forraje y las características morfogénicas y estructurales de Urochloa brizantha cv. Xaraés se evaluó em condiciones de campo. Aumentar el período dedescanso resultó en mayores rendimentos de matéria seca verde (MSV) y el vigor de rebrote, sin embargo, resultó en reducciones significativas de los contenidos de nitrogéno, fósforo, cálcio, magnésio y potássio. La longitude mediana de las hojas y sus tasas de aparición y de senescencia fueron directamente proporcionales a los períodos de descanso, mientras que lo contrário ocurrió para la tasa de aparición de hojas. Los mayores rendimientos de MSV, número de hojas por macollas, tasa de expansión de las hojas y índice de área foliar se obtuvieron a 40,8; 40,2; 26,2 y 48,2 dias de rebrote. El período de descanso más adecuado para el pastoreo de pasturas de $U$. brizantha $\mathrm{cv}$. Xaraés, destinada a conciliar la producción, el vigor de rebrote y la calidad del forraje, está entre 35 y 42 dias.

Palabras clave: composición química, hojas, macollaje, matéria seca, senescencia

\section{Introdução}

Em Roraima, a pecuária bovina é uma atividade econômica em franca expansão e as pastagens cultivadas representam o mais importante recurso forrageiro para a alimentação dos rebanhos. A utilização do pastejo contínuo ou períodos mínimos de descanso e altas intensidades de desfolhação são fatores que contribuem para baixa disponibilidade e qualidade da forragem, com reflexos negativos nos índices de desempenho zootécnico dos animais. A produtividade e a perenidade das pastagens decorrem, entre outros fatores, da capacidade de reconstituição e manutenção da área foliar após a desfolhação, a qual afeta a estrutura do dossel, determinando sua velocidade de crescimento, acúmulo de forragem, composição química e persistência (Nabinger and Pontes, 2002). O período de descanso disponibilizado para o crescimento da pastagem afeta sua produtividade, composição química, capacidade de rebrota e persistência. Pastejo mais frequente proporciona maior rendimento de forragem; porém, concomitantemente, ocorrem decréscimos acentuados em sua composição química, com maior acúmulo de material fibroso, decréscimo na relação folha/colmo e, consequentemente, menor consumo pelos animais (Costa, 2004; Lemaire et al., 2011).

No manejo adequado de pastagens busca-se o equilíbrio entre produção e qualidade da forragem, visando assegurar os requerimentos nutricionais dos animais e garantindo, simultaneamente, a persistência e a produtividade das pastagens. A produtividade das gramíneas forrageiras decorre da contínua emissão de folhas e perfilhos, processo que assegura a restauração da área foliar após o pastejo e a perenidade da pastagem. Os processos de formação e desenvolvimento de folhas são fundamentais para o crescimento vegetal, considerando-se a importância das folhas no processo de fotossíntese, ponto de partida para a formação de novos tecidos (Santos et al., 2012). A morfogênese de gramíneas durante seu crescimento vegetativo é caracterizada por três fatores: taxa de aparecimento, taxa de alongamento a longevidade das folhas. A taxa de aparecimento e a longevidade das folhas determinam o número de folhas vivas/perfilho, as quais são geneticamente determinadas e podem ser afetadas pelos fatores ambientais e as práticas de manejo adotadas (Nabinger and Pontes, 2002; Lemaire et al., 2011).

Neste trabalho foram avaliados os efeitos do período de descanso sobre a produção de forragem, vigor de rebrota, composição química e características morfogênicas e estruturais de Urochloa brizantha cv. Xaraés nos cerrados de Roraima.

\section{Material e Métodos}

$O$ ensaio foi conduzido no Campo Experimental da Embrapa Roraima, localizado em Boa Vista, durante o período de maio a setembro de 2014, o qual correspondeu a uma precipitação acumulada de $865,4 \mathrm{~mm}$ e temperatura média mensal de $24,86^{\circ} \mathrm{C}$. O solo da área experimental é um Latossolo Amarelo, textura média, com as seguintes características químicas, na profundidade de $0-20 \mathrm{~cm}: \mathrm{pH}_{\mathrm{H} 2 \mathrm{O}}=$ 4,$6 ; \mathrm{P}=2,1 \mathrm{mg} / \mathrm{kg} ; \mathrm{Ca}+\mathrm{Mg}=0,97 \mathrm{cmol}_{\mathrm{c}} \cdot \mathrm{dm}^{-3}$; $\mathrm{K}=0,04 \mathrm{cmol}_{\mathrm{c}} \cdot \mathrm{dm}^{-3} ; \mathrm{Al}=0,56 \mathrm{cmol}_{\mathrm{c}} \mathrm{dm}^{-3} ; \mathrm{H}+\mathrm{Al}=$ $2,62 \mathrm{cmol}_{\mathrm{c}} \cdot \mathrm{dm}^{-3}$.

O delineamento experimental foi inteiramente casualizado com três repetições. Os tratamentos consistiram de seis períodos de descanso $(14,21$, $28,35,42$ e 49 dias). A adubação de 
estabelecimento constou da aplicação de $50 \mathrm{~kg}$ de $\mathrm{P}_{2} \mathrm{O}_{5} \mathrm{ha}^{-1}$ e $60 \mathrm{~kg}$ de $\mathrm{K}_{2} \mathrm{O} \mathrm{ha}^{-1}$, sob a forma de superfosfato triplo e cloreto de potássio, respectivamente. O tamanho das parcelas foi de $2,0 \times 2,0 \mathrm{~m}$, sendo a área útil de $1,0 \mathrm{~m}^{2}$.

Os parâmetros avaliados foram rendimento de matéria seca verde (MSV), teores de nitrogênio $(\mathrm{N})$, fósforo $(\mathrm{P})$, cálcio $(\mathrm{Ca})$, magnésio $(\mathrm{Mg})$ e potássio $(\mathrm{K})$, número de folhas vivas perfilho ${ }^{-1}$ (NFV), taxa de aparecimento de folhas (TAF), taxa de expansão foliar (TEF), taxa de senescência foliar (TSF), comprimento médio de folhas (CMF) e índice de área foliar (IAF). A TEF e a TAF foram calculadas dividindo-se o comprimento acumulado de folhas e o número total de folhas no perfilho, respectivamente, pelo período de rebrota. O CMF foi determinado pela divisão do alongamento foliar total do perfilho pelo número de folhas. Para o cálculo da área foliar foram coletadas amostras de folhas verdes completamente expandidas, procurando-se obter uma área entre 200 e $300 \mathrm{~cm}^{2}$. As amostras foram digitalizadas e a área foliar estimada com o auxílio de planímetro ótico eletrônico (Li-Cor 3100C). Posteriormente, as amostras foram levadas à estufa com ar forçado a $65^{\circ} \mathrm{C}$ até atingirem peso constante, obtendo-se a MS foliar. A área foliar específica (AFE) foi determinada através da relação entre a área de folhas verdes e a sua MS $\left(\mathrm{m}^{2} / \mathrm{g}\right.$ MS foliar). O IAF foi determinado a partir do produto entre a MS total das folhas verdes $\left(\mathrm{g}\right.$ de $\left.\mathrm{MS} / \mathrm{m}^{2}\right)$ pela AFE $\left(\mathrm{m}^{2} / \mathrm{g}\right.$ de MS foliar). A TSF foi obtida dividindo-se o comprimento da folha que se apresentava de coloração amarelada ou necrosada pela idade de rebrota. O vigor de rebrota foi avaliado através da produção de MS aos 21 dias após o corte à idade do primeiro corte.

Os dados foram submetidos à análise de variância e de regressão considerando o nível de significância de $5 \%$ de probabilidade. Para se estimar a resposta dos parâmetros avaliados aos períodos de descanso, a escolha dos modelos de regressão baseou-se na significância dos coeficientes linear e quadrático, por meio do teste " $t$ ", de Student, ao nível de 5\% de probabilidade.

\section{Resultados e Discussão}

O período de descanso afetou os rendimentos de MSV $(\mathrm{P}<0,05)$, sendo a relação quadrática e o máximo valor estimado aos 40,8 dias de descanso (Tabela 1). Nos cerrados de Rondônia, para pastagens de Urochloa brizantha cv.
Marandu, Piatã e Xaraés, Costa et al. (2007) estimaram maiores rendimentos de forragem para períodos de descanso variando entre 28 e 35 dias. $\mathrm{O}$ efeito do período de descanso sobre o vigor de rebrota ajustou-se ao modelo quadrático de regressão e o máximo rendimento de MSV foi obtido aos 40,8 dias. Costa (2004) reportou máximo vigor de rebrota para pastagens de $U$. brizantha cv. Marandu no período entre 21 e 35 dias após o corte das plantas. A velocidade de rebrota apresenta alta correlação com a preservação dos meristemas apicais, pois com a sua preservação a formação de tecidos fotossintetizantes ocorre por meio da expansão de novas folhas, enquanto que com a remoção de meristemas apicais o novo crescimento depende do desenvolvimento de novas gemas, notadamente de origem basal, para a produção de folhas (Difante et al., 2011; Cunha et al., 2012). Os teores de N, P, Ca Mg e K foram negativa e linearmente afetados pelos períodos de descanso (Tabela 1), evidenciando um efeito de diluição de seus teores com o avanço do estádio de crescimento da gramínea. Os teores registrados neste trabalho foram semelhantes ou superiores aos relatados por Costa et al. (2007) para diversos genótipos de Urochloa, submetidos a diferentes períodos de descanso. Para o P, Ca e K, os teores obtidos com períodos de descanso de até 35 dias, foram superiores ao nível crítico interno determinado por (Costa, 2004) para U. brizantha cv. Xaraés $(1,71 ; 3,52$ e $17,06 \mathrm{~g} / \mathrm{kg}$, respectivamente para $\mathrm{P}, \mathrm{Ca}$ e $\mathrm{Mg}$ ).

A TAF foi negativa e linearmente afetada pelo período de descanso; enquanto que para o NFV, TEF, CMF e IAF os ajustes foram quadráticos e os máximos valores obtidos aos 40,$2 ; 26,2 ; 38,1$ e 48,2 dias de descanso, respectivamente (Tabela 2). Nos cerrados de Rondônia, em pastagens de Urochloa humidicola, $U$. dictyoneura e $U$. brizantha cv. Marandu, Costa (2004) constatou maiores NFV, TEF e IAF para períodos compreendidos entre 28 e 35 dias de rebrota, os quais foram recomendados como mais adequados para o manejo das gramíneas. O IAF representa a síntese das características morfogênicas e estruturais da gramínea e reflete o balanço dos processos que determinam a oferta (fotossíntese) e a demanda (respiração, acúmulo de reservas, síntese e senescência de tecidos) de fotoassimilados, estabelecendo o ritmo de crescimento da pastagem (Nabinger and Carvalho, 2009). Com o aumento do período de descanso, a percentagem de luz interceptada pelo dossel 
atinge seu ponto máximo, $\mathrm{IAF}_{\text {teto, }}$ onde para cada nova folha surgida na porção superior da planta ocorre a senescência de uma folha em sua porção inferior, o que estabiliza ou reduz a disponibilidade de biomassa verde como decorrência da redução do coeficiente de extinção luminosa (Nabinger and Pontes, 2002).

Tabela 1. Produção de matéria seca verde (MSV - $\left.\mathrm{kg} \mathrm{ha}^{-1}\right)$, vigor de rebrota (VR - $\mathrm{kg} \mathrm{MSV} / 21$ dias) e teores de nitrogênio $(\mathrm{N})$, fósforo $(\mathrm{P})$, cálcio $(\mathrm{Ca})$, magnésio $(\mathrm{Mg})$ e potássio $(\mathrm{K})$ de Urochloa brizantha cv. Xaraés, em função do período de descanso.

\begin{tabular}{cccccccc}
\hline \multirow{2}{*}{ Variáveis } & \multicolumn{6}{c}{ Períodos de descanso (días) } & \multirow{2}{*}{ Equação de Regressão } \\
\cline { 2 - 7 } & 14 & 21 & 28 & 35 & 42 & 49 & \\
\hline $\mathrm{MSV}$ & 1.315 & 1.764 & 2.318 & 2.657 & 2.819 & 2.472 & $\mathrm{Y}=687+165,29 \mathrm{X}-2,0219 \mathrm{X}^{2}\left(\mathrm{R}^{2}=0,91\right)$ \\
$\mathrm{VR}$ & 935 & 1307 & 1611 & 1835 & 1922 & 1721 & $\mathrm{Y}=371+110,19 \mathrm{X}-1,3604 \mathrm{X}^{2}\left(\mathrm{R}^{2}=0,93\right)$ \\
$\mathrm{N}(\mathrm{g} / \mathrm{kg})$ & 27,54 & 25,11 & 24,87 & 24,03 & 22,99 & 21,33 & $\mathrm{Y}=29,23-0,1561 \times\left(\mathrm{r}^{2}=0,86\right)$ \\
$\mathrm{P}(\mathrm{g} / \mathrm{kg})$ & 1,97 & 1,92 & 1,83 & 1,74 & 1,71 & 1,63 & $\mathrm{Y}=2,111-0,0099 \times\left(\mathrm{r}^{2}=0,95\right)$ \\
$\mathrm{Ca}(\mathrm{g} / \mathrm{kg})$ & 4,29 & 4,02 & 3,89 & 3,71 & 3,66 & 3,52 & $\mathrm{Y}=4,505-0,0209 \times\left(\mathrm{r}^{2}=0,93\right)$ \\
$\mathrm{Mg}(\mathrm{g} / \mathrm{kg})$ & 3,39 & 3,07 & 2,88 & 2,71 & 2,65 & 2,49 & $\mathrm{Y}=3,627-0,0242 \times\left(\mathrm{r}^{2}=0,95\right)$ \\
$\mathrm{K}(\mathrm{g} / \mathrm{kg})$ & 20,12 & 19,32 & 18,51 & 17,33 & 16,59 & 16,02 & $\mathrm{Y}=21,822-0,1219 \times\left(\mathrm{r}^{2}=0,98\right)$ \\
\hline
\end{tabular}

O CMF é uma característica plástica, responsiva à intensidade e frequência de desfolhação e considerada a principal estratégia morfológica de escape das plantas ao pastejo (Pedreira et al., 2009; Lemaire et al., 2011). As gramíneas sob desfolhações frequentes podem apresentar abundante perfilhamento, hábito de crescimento prostrado e elevado ritmo de expansão foliar, possibilitando maior interceptação de luz e rebrotas mais vigorosas (Nabinger and Pontes, 2002). As TAF, TEF e CMF obtidas neste trabalho, independentemente dos períodos de descanso, foram superiores às reportados por Difante et al. (2011) avaliando $U$. brizantha cv. Marandu, sob diferentes intervalos entre cortes, que estimaram valores médios de 0,083 folhas $^{-1}$ perfilho $^{-1}$ dia; $1,61 \mathrm{~cm} \mathrm{dia}{ }^{-1}$ perfilho $^{-1}$ e $18,59 \mathrm{~cm}$ para o tamanho médio de folhas. O NFV, constante a partir do momento em que a TSF se iguala a TAF, constitui critério objetivo e prático para a definição dos períodos de descanso na lotação rotativa e a intensidade de pastejo na lotação contínua (Costa et al., 2014). Para pastagens de $U$. brizantha cv. Marandu, Costa (2004) recomenda o início do pastejo quando os perfilhos apresentarem, em média, 4 a 5 folhas vivas, de modo a maximizar a produtividade e a qualidade da forragem.

Tabela 2. Número de folhas vivas perfilho ${ }^{-1}$ (NFV), taxa de aparecimento de folhas (TAF- folhas dia $^{-1}$ perfilho ${ }^{-1}$ ), taxa de expansão foliar (TEF - $\mathrm{cm} \mathrm{dia}^{-1}$ perfilho ${ }^{-1}$ ), comprimento médio de folhas (CMF $\mathrm{cm}$ ), índice de área foliar (IAF) e taxa de senescência foliar (TSF - cm dia ${ }^{-1}$ perfilho ${ }^{-1}$ ) de Urochloa brizantha cv. Xaraés, em função do período de descanso.

\begin{tabular}{cccccccc}
\hline \multirow{2}{*}{ Variáveis } & \multicolumn{6}{c}{ Períodos de descanso (días) } & \multirow{2}{*}{ Equação de Regressão } \\
\cline { 2 - 7 } & 14 & 21 & 28 & 35 & 42 & 49 & \\
\hline NFV & 2,98 & 3,77 & 4,61 & 5,23 & 5,46 & 4,88 & $\mathrm{Y}=0,3401+0,2753 \mathrm{X}-0,0034 \mathrm{X}^{2}\left(\mathrm{R}^{2}=0,91\right)$ \\
TAF & 0,213 & 0,180 & 0,165 & 0,149 & 0,131 & 0,108 & $\mathrm{Y}=0,2499-0,0031 \times\left(\mathrm{r}^{2}=0,97\right)$ \\
TEF & 3,150 & 3,285 & 3,441 & 3,362 & 2,821 & 2,071 & $\mathrm{Y}=1,673+0,1375 \mathrm{X}-0,0026 \mathrm{X}^{2}\left(\mathrm{R}^{2}=0,93\right)$ \\
CMF & 14,8 & 18,3 & 20,9 & 22,5 & 21,7 & 20,8 & $\mathrm{Y}=3,44+0,9881 \mathrm{X}-0,0132 \mathrm{X}^{2}\left(\mathrm{R}^{2}=0,89\right)$ \\
IAF & 0,81 & 1,43 & 2,12 & 2,97 & 3,19 & 3,02 & $\mathrm{Y}=1,7567+0,2041 \mathrm{X}-0,0021 \mathrm{X}^{2}\left(\mathrm{R}^{2}=0,95\right)$ \\
TSF & 0,098 & 0,131 & 0,149 & 0,183 & 0,201 & 0,237 & $\mathrm{Y}=0,0458+0,00381 \times\left(\mathrm{r}^{2}=0,95\right)$ \\
\hline
\end{tabular}

A TEF, em decorrência de sua alta correlação 
com a produção de MS, tem sido utilizada como critério para a seleção de gramíneas em trabalhos de melhoramento genético (Nabinger and Carvalho, 2009). A TEF está diretamente correlacionada com o CMF e, folhas menores estão normalmente associadas a maiores valores de TAF. No presente trabalho, a correlação entre TEF e rendimento de MS foi positiva e significativa $(r=0,886 ; \mathrm{P}<0,05)$; enquanto que com a TAF a correlação foi negativa e não significativa $(\mathrm{r}=-0,587 ; \mathrm{P}>0,05)$. A TEF explicou em $78,4 \%$ os incrementos verificados nos rendimentos de MSV, em função do período de descanso. A TAF é o componente morfogênico de maior destaque, uma vez que afeta diretamente as três características estruturais do relvado: tamanho da folha, densidade de perfilhos e número de folhas perfilho ${ }^{-1}$ (Pedreira et al., 2009, Santos et al., 2012). Para Difante et al. (2011), a TAF e a TEF apresentam geralmente correlação negativa, indicando que quanto maior a TAF, menor será o tempo disponível para o alongamento foliar (Nabinger and Carvalho, 2009).

A TSF foi direta e linearmente proporcional ao período de descanso (Tabela 2). A senescência foliar é decorrente da competição por metabólitos e nutrientes entre as folhas velhas e as jovens em crescimento, o que ocasiona redução da disponibilidade de forragem de boa qualidade (Lemaire et al., 2011). Os valores registrados foram inferiores aos reportados por Costa (2004) para $U$. brizantha cv. Marandu $(0,162 ; 0,193$ e $0,206 \mathrm{~cm} \mathrm{dia}^{-1}$ perfilho- $^{-1}$, respectivamente para períodos de descanso de 21,35 e 42 dias). A senescência é um processo natural caracterizando a última fase de desenvolvimento de uma folha, iniciado após completa expansão das primeiras folhas e acentuado progressivamente com o aumento da área foliar, em decorrência do sombreamento das folhas inseridas na porção inferior e do baixo suprimento de radiação fotossinteticamente ativa, além de forte competição por luz, nutrientes e água entre os diversos estratos da planta (Nabinger and Pontes, 2002). Quando o perfilho atinge determinado NFV ocorre equilíbrio entre a TAF e a senescência das folhas que ultrapassaram seu período de duração de vida, de modo que o surgimento de nova folha implica na senescência da folha que a precedeu, mantendo o NFV relativamente constante (Lemaire et al., 2011). A senescência apesar de afetar negativamente a qualidade da forragem representa importante processo fisiológico no fluxo de tecidos da gramínea, pois em torno de $35 ; 68 ; 86$ e $42 \%$ do nitrogênio, fósforo, potássio e magnésio, respectivamente, podem ser reciclados das folhas senescentes e utilizados para a produção de novos tecidos foliares (Sarmiento et al., 2006, Costa et al., 2013).

\section{Conclusões}

O aumento do período de descanso favorece os rendimentos de forragem e o vigor de rebrota, contudo reduz os teores de $\mathrm{N}, \mathrm{P}, \mathrm{Ca}, \mathrm{Mg}$ e $\mathrm{K}$. O comprimento médio de folhas e suas taxas de expansão e senescência são diretamente proporcionais aos períodos de descanso, ocorrendo o inverso quanto à taxa de aparecimento foliar. O período de descanso mais adequado para pastagens de $U$. brizantha $\mathrm{cv}$. Xaraés, visando a conciliar produção, vigor de rebrota e qualidade da forragem, situa-se entre 35 e 42 dias.

\section{Referências Bibliográficas}

Costa, N. L. 2004. Formação, manejo e recuperação de pastagens em Rondônia. Porto Velho: Embrapa Rondônia, 215p.

Costa, N. L., Magalhães, J. A., Pereira, R. G. A., Townsend, C. R. \& Oliveira, J. R. C. 2007. Considerações sobre o manejo de pastagens na Amazônia Ocidental. Revista do Conselho Federal de Medicina Veterinária, 40, 37-56.

Costa, N. L., Moraes, A., Carvalho, P. C. F., Monteiro, A. L. G., Motta, A. C. V., Silva, A. L. P. \& Oliveira, R. A. 2014. Morfogênese de Trachypogon plumosus sob calagem, adubação e idades de rebrota. Archivos de Zootecnia, 63, 109-120.

Costa, N. L., Moraes, A., Monteiro, A. L. G., Motta, A. C. V., Oliveira, R. A. \& Rodrigues, A. N. A. 2013. Forage productivity and morphogenesis of Axonopus aureus under different nitrogen fertilization rates. Revista Brasileira de Zootecnia, 42, 541-548.

Cunha, F. F., Mota Ramos, M., Brasileiro, A. C. A., Alves , O., Rubens, Cóser, A. C., Martins, C. E., Cecon, P. R. \& Silva, A. R. A. 2012. Produtividade da Brachiaria brizantha cv. Xaraés em diferentes manejos e doses de adubação, períodos de descanso e épocas do ano. Idesia (Arica), 30, 75-82.

Difante, G. S., Nascimento Júnior, D., Silva, S. C., Euclides, V. P. B. \& Montagner, D. B. 2011. Características morfogênicas e 
estruturais do capim-marandu submetido a combinações de alturas e intervalos de corte. Revista Brasileira de Zootecnia, 40, 955-963.

Lemaire, G., Hodgson, J. \& Chabbi, A. 2011. Grassland productivity and ecosystem services. Cabi, Wallingford.

Nabinger, C. \& Carvalho, P. F. C. 2009. Ecofisiología de sistemas pastoriles: aplicaciones para su sustentabilidad. Agrociencia, 13, 18-27.

Nabinger, C. \& Pontes, L. S. 2002. Manejo da desfolha. Simpósio sobre manejo da pastagem. FEALQ, Jaboticabal. p.192-210.

Pedreira, B., Pedreira, C. G. S. \& Silva, S. C. 2009. Acúmulo de forragem durante a rebrotação de capim-xaraés submetido a três estratégias de desfolhação. Revista Brasileira de Zootecnia, 38, 618-625.

Santos, M. R., Fonseca, D. M., Gomes, V. M., Silva, S. P., Silva, G. P. \& Reis, M. 2012. Correlações entre características morfogênicas e estruturais em pastos de capim-braquiária. Ciência Animal Brasileira, 13, 49-56.

Sarmiento, G., Silva, M. P., Naranjo, M. E. \& Pinillos, M. 2006. Nitrogen and phosphorus as limiting factors for growth and primary production in a flooded savanna in the Venezuelan Llanos. Journal of Tropical Ecology, 22, 203-212.

\section{Article History:}

Received 8 February 2017

Accepted 26 March 2017

Available on line 4 May 2017

License information: This is an open-access article distributed under the terms of the Creative Commons Attribution License 4.0, which permits unrestricted use, distribution, and reproduction in any medium, provided the original work is properly cited. 